\title{
The Effects of Phase Advances between Interaction Points
}

\author{
Y. Luo, S. Tepekian, W. Fischer, X. Gu, D. Trbojevic
}

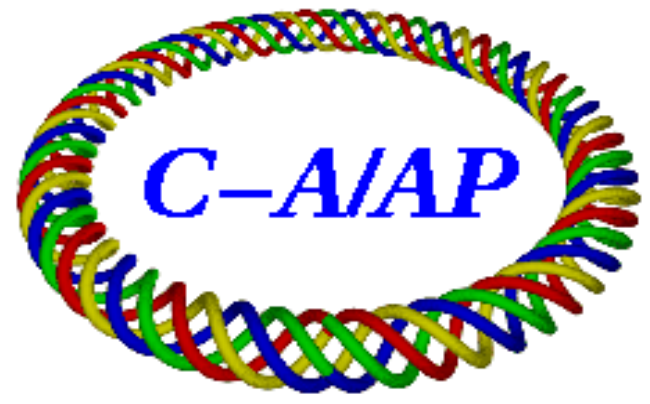

\section{Collider-Accelerator Department Brookhaven National Laboratory Upton, NY 11973}

Notice: This document has been authorized by employees of Brookhaven Science Associates, LLC under Contract No. DE-AC02-98CH10886 with the U.S. Department of Energy. The United States Government retains a nonexclusive, paid-up, irrevocable, world-wide license to publish or reproduce the published form of this document, or allow others to do so, for United States Government purposes. 
March 8, 2011

\title{
The effects of phase advances between interaction points
}

\author{
Y. Luo, S. Tepekian, W. Fischer, X. Gu, D. Trbojevic \\ Brookhaven National Laboratory, Upton, NY 11973, USA
}

In this note we perform simulation studies to investigate the effects of betatron phase advances between the beam-beam interaction points on half-integer resonance driving term, second order chromaticty and dynamic aperture in RHIC. The betatron phase advances are adjusted with artificial matrices inserted in the middle of arcs. The lattices for the 2011 RHIC polarized proton (p-p) run and 2010 RHIC Au-Au runs are used. We also scan the phase advances between IP8 and the electron lens for the proposed Blue ring lattice with head-on beam-beam compensation.

\section{Introduction}

According to Ref. [1], the triplet quadruples in the interaction regions (IRs) IR6 and IR8 contribute most of the second order chromaticities for the RHIC lattices. The reason is that $\beta$ functions in the triplets are very big due to the low $\beta^{*}$ at the interaction points(IPs) IP6 and IP8.

According to Ref. [2], the second order chromaticities are given by

$$
\xi_{x, y}^{(2)}=-\frac{1}{2} \xi_{x, y}^{(1)}+\frac{1}{8 \pi} \oint\left[\mp K_{1} \pm K_{2} D_{x}\right] \frac{\partial \beta_{x, y}}{\partial \delta} d s+\frac{1}{8 \pi} \oint \pm K_{2} \beta_{x, y} D_{x}^{(2)} d s,
$$

where $K_{1}$ and $K_{2}$ are the strengths of quadrupoles and sextupoles. $\xi_{x, y}^{(1)}$ are the first order chromaticities. $\beta_{x, y}$ are betatron amplitude functions. $D_{x}$ and $D_{x}^{(2)}$ are the first order and second order horizontal dispersion. $\delta$ is the relative momentum deviation.

The second term in Eq. (1) is the dominant one which is determined by the off-momentum $\beta$-beat $\frac{\partial \beta_{x, y}}{\partial \delta}$. The relative off-momentum $\beta$-beat is

$$
\begin{aligned}
& \frac{1}{\beta_{x, y}(s)} \frac{\partial \beta_{x, y}(s)}{\partial \delta}= \\
& \pm \frac{1}{2 \sin \left(2 \pi Q_{x, y}\right)} \oint \beta_{x, y}\left(s^{\prime}\right)\left[\mp K_{1}\left(s^{\prime}\right) \pm K_{2}\left(s^{\prime}\right) D_{x}\left(s^{\prime}\right)\right] \cos \left(2\left|\phi_{x, y}(s)-\phi_{x, y}\left(s^{\prime}\right)\right|-2 \pi Q_{x, y}\right) d s^{\prime} .
\end{aligned}
$$

We define half integer resonance driving terms as

$$
\begin{aligned}
& h_{20001}=\sum_{i}^{N}\left[-\left(K_{1} L\right)_{i}+\left(K_{2} D_{x} L\right)_{i}\right] \beta_{x, i} e^{-i 2 \phi_{x, i}}, \\
& h_{00201}=\sum_{i}^{N}\left[+\left(K_{1} L\right)_{i}-\left(K_{2} D_{x} L\right)_{i}\right] \beta_{y, i} e^{-i 2 \phi_{y, i}} .
\end{aligned}
$$

Here $h_{20001}$ and $h_{00201}$ are the horizontal and vertical half integer resonance driving terms. Comparing Eqs. (3) and (4) to Eq. (2), the relative off-momentum $\beta$-beat is governed by the half integer resonance driving terms.

Therefore, minimizing the contributions from the triplets from IR6 and IR 8 to the half integer resonance driving terms will reduce off-momentum $\beta$-beat and second order chromaticities. In the following we perform simulation studies to scan the betatron phase advances between IP6 and IP8 to investigate their effects on the second order chromaticities and dynamic aperture.

\section{Adjustments of phase advances between IPs}

\subsection{RHIC Facts}

The RHIC ring lattices have more or less three fold symmetry, with three outer arcs and three inner arcs. Between adjacent arcs are interaction regions. Each arc consists of 11 FODO cells. In RHIC, all the focusing 
Table 1: Tunes and chromaticities

\begin{tabular}{lcccc}
\hline \hline Lattice & $(\mathrm{Qx} \mathrm{Qy})$ & $\left(\xi_{x}^{(1)}, \xi_{y}^{(1)}\right)$ & $\left(\xi_{x}^{(2)}, \xi_{y}^{(2)}\right)$ & $\left(\xi_{x}^{(3)}, \xi_{y}^{(3)}\right)$ \\
\hline 2011pp-Blue & $(28.675,29.68)$ & $(0.93,1.00)$ & $(2241.08,4027.64)$ & $(748942,-67422)$ \\
2011pp-Yellow & $(28.675,29.68)$ & $(0.99,0.88)$ & $(-6308.92,-1123.51)$ & $(13825,1275500)$ \\
2010Au-Blue & $(31.23,32.22)$ & $(1.09,0.99)$ & $(-4929.59,-1021.05)$ & $(-1020090,5850.7)$ \\
2010Au-Yellow & $(31.23,32.22)$ & $(0.94,0.95)$ & $(3736.88,1683.07)$ & $(650640,447817)$ \\
elens-Blue & $(28.675,29.68)$ & $(0.93,1.04)$ & $(2540.68,2733.72)$ & $(736772,-449500)$ \\
\hline \hline
\end{tabular}

Table 2: $\beta$-functions at IPs

\begin{tabular}{lcccccc}
\hline \hline Lattice & IP6 & IP8 & IP10 & IP12 & IP2 & IP4 \\
\hline 2011pp-Blue & $(0.63,0.64)$ & $(0.62,0.64)$ & $(7.60,7.72)$ & $(7.48,7.72)$ & $(3.02,3.06)$ & $(7.47,7.65)$ \\
2011pp-Yellow & $(0.63,0.66)$ & $(0.62,0.66)$ & $(6.66,6.61)$ & $(6.53,6.56)$ & $(3.00,3.05)$ & $(6.53,6.67)$ \\
2010Au-Blue & $(0.67,0.69)$ & $(0.67,0.69)$ & $(4.14,4.04)$ & $(3.96,3.95)$ & $(3.96,3.95)$ & $(4.14,4.04)$ \\
2010Au-Yellow & $(0.76,0.68)$ & $(0.76,0.68)$ & $(3.77,4.06)$ & $(3.94,4.01)$ & $(3.94,4.01)$ & $(3.77,4.06)$ \\
elens-Blue & $(0.53,0.53)$ & $(0.53,0.53)$ & $(10.18,9.96)$ & $(9.92,9.98)$ & $(9.92,9.98)$ & $(10.1,9.96)$ \\
\hline \hline
\end{tabular}

Table 3: Betatron phase advances ( in unit of $\pi$ ) between IPs

\begin{tabular}{lcccccc}
\hline \hline Lattice & (IP6-IP8) & (IP8-IP10) & (IP10-IP12) & (IP12-IP2) & (IP2-IP4) & (IP4-IP6) \\
\hline 2011pp-Blue & $(10.65,8.64)$ & $(8.45,10.98)$ & $(10.68,8.92)$ & $(8.52,10.94)$ & $(10.58,8.87)$ & $(8.45,10.98)$ \\
2011pp-Yellow & $(8.32,11.03)$ & $(10.67,8.80)$ & $(8.57,10.90)$ & $(10.58,8.88)$ & $(8.51,10.92)$ & $(10.67,8.80)$ \\
2010Au-Blue & $(11.41,9.44)$ & $(9.10,11.92)$ & $(11.84,9.53)$ & $(9.15,12.07)$ & $(11.84,9.53)$ & $(9.10,11.92)$ \\
2010Au-Yellow & $(9.04,11.77)$ & $(11.62,9.48)$ & $(9.16,12.07)$ & $(11.83,9.54)$ & $(9.16,12.07)$ & $(11.62,9.48)$ \\
elens-Blue & $(10.61,8.56)$ & $(8.41,10.96)$ & $(10.66,8.91)$ & $(8.56,11.02)$ & $(10.66,8.91)$ & $(8.41,10.96)$ \\
\hline \hline
\end{tabular}

and all the defocusing quadrupole magnets in the arcs are powered by the same power supplies. So far it is not possible to adjust individual arc's phase advances with quadrupoles in FODO cells. Considering the three-fold symmetry of RHIC rings, the betatron phase advances in the 3 outer or in the 3 inner rings are more or less the same.

To adjust the betatron phase advance between IP6 and IP8, we should adjust the betatron phase advances for all the outer and inner rings at same time. To keep the tunes constant, if we increase or decrease the phase advances of all outer rings, we will decrease or increase same amounts of betatron phase advances in all inner rings.

\subsection{Simulation Approach}

To avoid real lattice matching, in this simulation study we adjust the phase advances by inserting artificial phase shifting matrices in the centers of arcs. The phase shifting matrices will not affect tunes, Twiss parameters and dispersion functions. Again, to keep the tunes constant, if the phase shifting matrices in the outter rings increase or decrease the phase advances, the phase shifting matrices in the inner rings will decrease or increase the same amount.

Comparing the real lattices and the above 'fake' simulation study lattices, the phase advances between IPs and nonlinear field errors in the IRs are the same although the phase advances of FODO cells are different. As we know, the dynamic aperture is mainly determined by the nonlinear field errors and beambeam interactions for RHIC lattices. Therefore, the results from our simulation study with artificial phase shifting matrices will reflect most of the physics with the real lattices.

In this study we adopt the lattices for the 2011 RHIC polarized proton (p-p) run and $2010 \mathrm{RHIC} \mathrm{Au}-$ $\mathrm{Au}$ runs, and the Blue ring lattice for head-on beam-beam compensation. For the head-on beam-beam compensation lattice, we will study the effects of phase advance between IP8 and e-lens which is located $1.0 \mathrm{~m}$ away from IP10.

For each lattice, we will scan the horizontal and vertical phase advances between IPs. For each step, we calculate the absolute values of half integer resonance driving terms only from the triplets in the IR6 and IR8 to check the cancellation between IR6 and IR8. The reference point of resonance driving terms is IP6. We also calculate second order chromaticities and dynamic apertures (DAs). The second order 
chromaticities change in the scan because of the betatron phase changes between IRs. The phase shifting matrices themselves have no contributions to chromaticities.

\subsection{Lattice and beam parameters}

Table 1-3 list the tunes, chromaticities, $\beta$ at IPs, and phase advances betwen IPs for all the lattices without phase advance adjustment between IPs.

DAs are calculated in $10^{6}$ turn trackings. The tunes of zero-amplitude particles are set to $(28.675,29.68)$ for proton run and $(31.23,32.22)$ for $\mathrm{Au}-\mathrm{Au}$ run . These tunes are with beam-beam interaction at IP6 and IP8. The first order chromaticities are set to $(1,1)$. The initial relative momentum deviation is 0.0005 for protons and 0.0017 for $\mathrm{Au}$ ions. The $\mathrm{DA}$ for $\mathrm{Au}-\mathrm{Au}$ run is after RF re-bucketing. The proton bunch intensities for the $2011 \mathrm{p}-\mathrm{p}$ run lattices is $1.5 \times 10^{11}$. The $\mathrm{Au}$ ion bunch intensities for the $2010 \mathrm{Au}-\mathrm{Au}$ run lattices is $1.0 \times 10^{9}$. The proton bunch intensity for the head-on beam-beam compensation test is $2.5 \times 10^{11}$. Half beam-beam compensation is included in this lattice.

\section{Results}

\subsection{1 p-p run lattices}

Figures 1-3 and Figure 4-6 show the calculated horizontal half integer resonance term $h 20001$ and vertical half integer resonance term $h 00201$ from the triplets in IR6 and IR, second chromaticities and DAs for the BLue ring and Yellow ring lattices. The axis $\Delta \Phi_{x, y}$ are the phase advances between IP6 and IP8.

For Blue ring, the minimum of $h 20001$ is around $\Delta \Phi_{x}=10.6 \pi$ and the minimum of $h 20001$ is around $\Delta \Phi_{y}=8.7 \pi$. The minimum of horizontal second order chromaticity occurs around $\Delta \Phi_{x}=10.4 \pi$ and $\Delta \Phi_{x}=$ $10.8 \pi$. The minimum of vertical second order chromaticity occurs around $\Delta \Phi_{x}=8.5 \pi$ and $\Delta \Phi_{x}=9.0 \pi$.

For Yellow ring, the minimum of $h 20001$ is around $\Delta \Phi_{x}=8.7 \pi$ and the minimum of $h 20001$ is around $\Delta \Phi_{y}=10.6 \pi$. The minimum of horizontal second order chromaticity occurs around $\Delta \Phi_{x}=8.5 \pi$. The minimum of vertical second order chromaticity occurs around $\Delta \Phi_{y}=11.0 \pi$ and $11.5 \pi$.

Figure 3 and Figure 6 show the DAs for the Blue and Yellow rings in the phase advance scan. For the Blue ring, the maximum DA is around $\left(\Delta \Phi_{x}=10.65 \pi, \Delta \Phi_{y}=8.5 \pi\right)$. For the Yellow ring, the maximum DA is around $\left(\Delta \Phi_{x}=8.8 \pi, \Delta \Phi_{y}=10.9 \pi\right)$.

The phase advances without phase adjustment between IP6 and IP8 are $(10.65 \pi, 8.64 \pi)$ for the Blue ring and $(8.32 \pi, 11.03 \pi)$ for the Yellow ring. Therefore, the phase advances between IP6 and IP8 without phase adjustment are in the good DA region for Blue ring. For the Yellow ring, the default phase advances between IP6 and IP8 are not in the good DA region.

\section{$3.22010 \mathrm{Au}-\mathrm{Au}$ run lattices}

Figures 7-9 and Figures 10-12 plot the half integer resonance terms, second order chromaticities and DAs for the Blue and Yellow ring lattices for $2010 \mathrm{Au}-\mathrm{Au}$ run lattices.

For the Blue ring, the minimum of horizontal and vertical half integer resonance driving terms occur at $\Delta \Phi_{x}=10.6$ and $\Delta \Phi_{y}=9.75$. The minimum of horizontal and vertical second order chromaticities happen at $\Delta \Phi_{x}=11.55$ and $\Delta \Phi_{y}=9.5$. In this scan, the DA is mostly sensitive to the horizontal phase advance between IP6 and IP8. The good region of DA is around $\left(\Delta \Phi_{x}=10.6 \pi\right.$ or $\left.11.0 \pi, \Delta \Phi_{y}=(9.4-9.8) \pi\right)$.

The Yellow ring, the minimum of horizontal and vertical half integer resonance driving terms happen at $\Delta \Phi_{x}=8.8$ and $\Delta \Phi_{y}=11.65$. The minimum of horizontal and vertical second order chromaticities happen at $\Delta \Phi_{x}=9.15$ and $\Delta \Phi_{y}=11.45$. The good DA region is around $\left(\Delta \Phi_{x}=(9.2-9.5) \pi\right.$ and $\left.\Delta \Phi_{y}=(11.5-11.7) \pi\right)$.

The phase advances without phase adjustment between IP6 and IP8 are $(11.41 \pi, 9.44 \pi)$ for the Blue ring and $(9.04 \pi, 11.77 \pi)$ for the Yellow ring. The phase advances between IP6 and IP8 without phase adjustment are not in good DA region for both rings.

\subsection{Beam-beam compensation lattice}

Figures 13 and 14 plot second order chromaticities and DA for the proposed head-on beam-beam compensation Blue ring lattice versus the phase advances between IP 8 and the center of e-lens. In these plots, the phase advances $\Delta \Phi_{x}$ and $\Delta \Phi_{y}$ are between IP8 and the center of the e-lens. 
From Figures 13, the minimum of horizontal and vertical half integer resonance driving terms happen at $\Delta \Phi_{x}=8.35$ and $\Delta \Phi_{y}=11.07$. From Figures 14 , the maximum DA occurs at $\left(\Delta \Phi_{x}=8.65 \pi, \Delta \Phi_{x}=10.85 \pi\right)$. There are a large good DA region between $\left(\Delta \Phi_{x}=(8.1-8.9) \pi\right.$ and $\left.\Delta \Phi_{y}=(10.7-11.1) \pi\right)$.

The default phase advances between IP8 and the e-lens center is $(8.5 \pi, 11.0 \pi$. In the previous simulation study, for better beam-beam compensation, we adjusted the phase advances between IP8 and the center of e-lens to $(9.0 \pi, 11.0 \pi)$ which is away from the good DA region shown in Figure 14.

\section{Discussion}

In this note we calculated half integer resonance driving terms, second order chromaticities and DA in the scan of phase advances between IP6 and IP8. Our goal is to find out the optimum betatron phase advances between IPs which give smaller second order chromaticities and bigger DAs. Smaller second order chromaticities are very important for the Au-Au run where RF-rebucketing increases the off-momentum deviation.

From the above studies, the phase advances for the minimum half integer resonance driving terms and for the minimum second order chromaticities do not always coincide. This may be explained by the fact that half integer resonance driving terms are calculated only from the triplets in IR6 and IR8. The contributions from other quadrupoles and sextupoles are excluded since we do not have their exact phase advances with artificial phase shifting matrices.

From second order chromaticities and DA calculation, we always find that good DA area in the $\left(\Delta \Phi_{x}, \Phi_{y}\right)$ plane always have small second order chromatities. For low $\beta^{*}$ lattices, it is very important to compensate the large second order chromaticities. From our study, it is possible to adjust the phase advances between IPs to minimize the triplets' contributions to second order chromaticities.

In the above study we fixed the tunes unchanged during the scan of phase advances between IPs. We also need to do tune scan to find out their best settings to maximize the dynamic aperture. The tunes used in this study are close to their values in the RHIC operations.

To confirm the optimum betatron phase advances between IP6 and IP 8 found in this study, we need to generate real RHIC lattices and calculate second order chromaticities and DA again. This work will be discussed in the coming RHIC summer shutdown.

\section{References}

[1] Y. Luo, et al, BNL RHIC/AP/418, January, 2011.

[2] Y. Luo, et al, BNL RHIC/AP/348, January, 2009. 

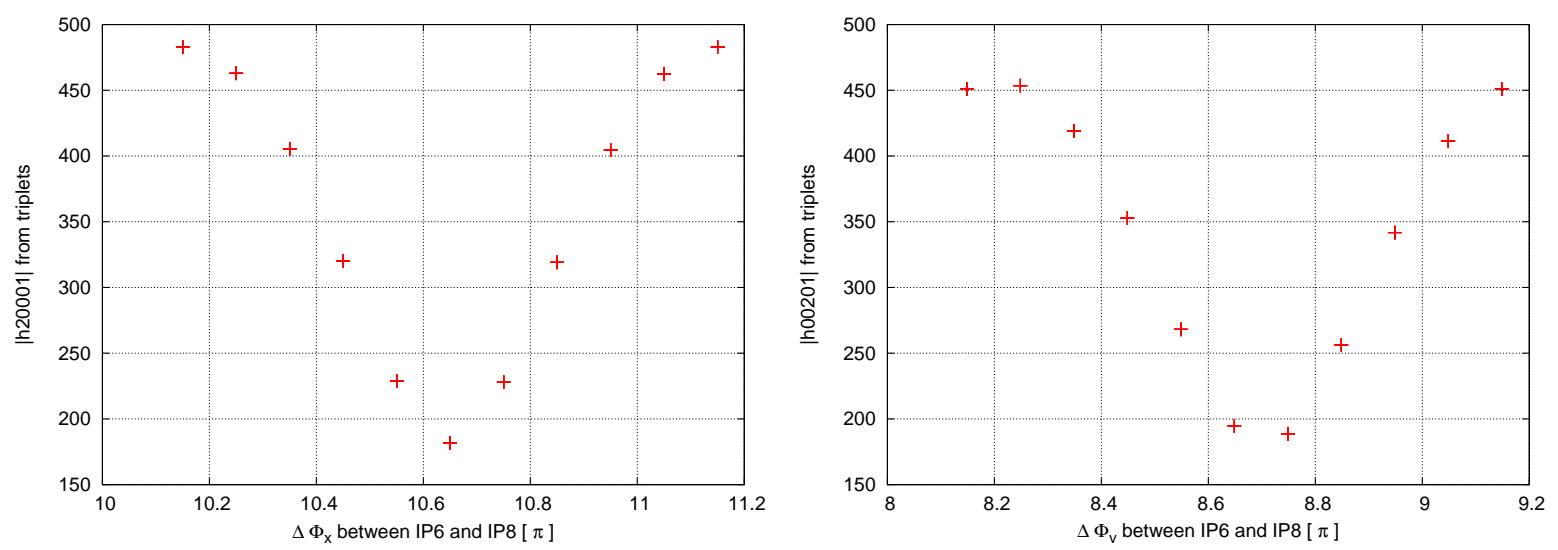

Figure 1: 2011pp-Blue: RDT versus phase advances between IP6 and IP8.
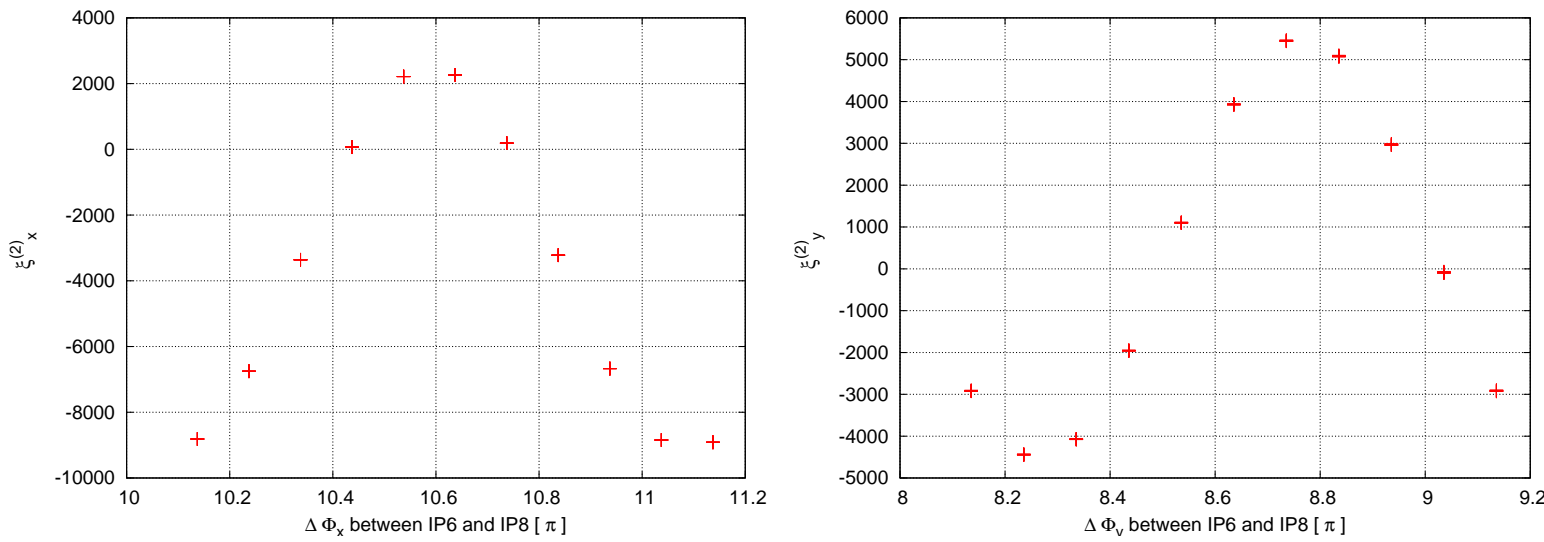

Figure 2: 2011pp-Blue: second order chromaticities versus phase advances between IP6 and IP8.

\section{Dynamic Aperture $[\sigma]$}

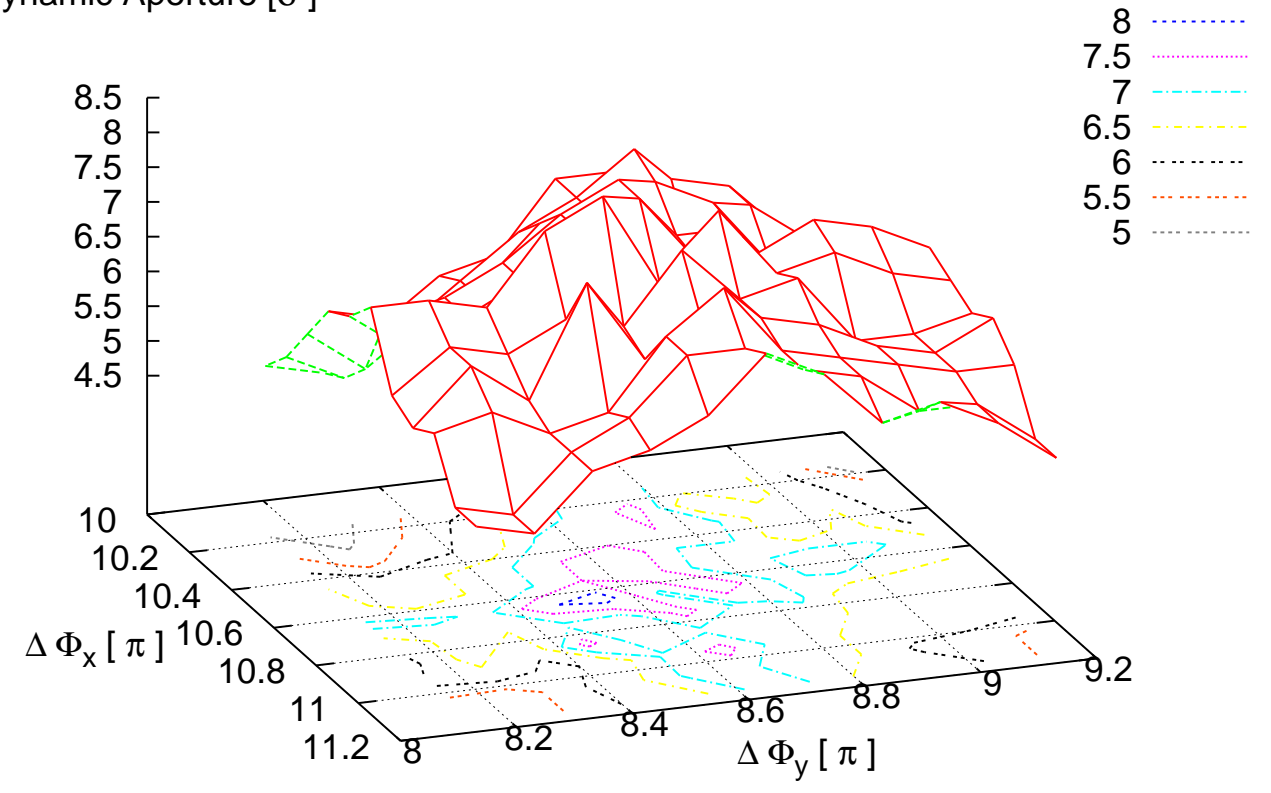

Figure 3: 2011pp-Blue: Dynamic aperture versus phase advances between IP6 and IP8. 

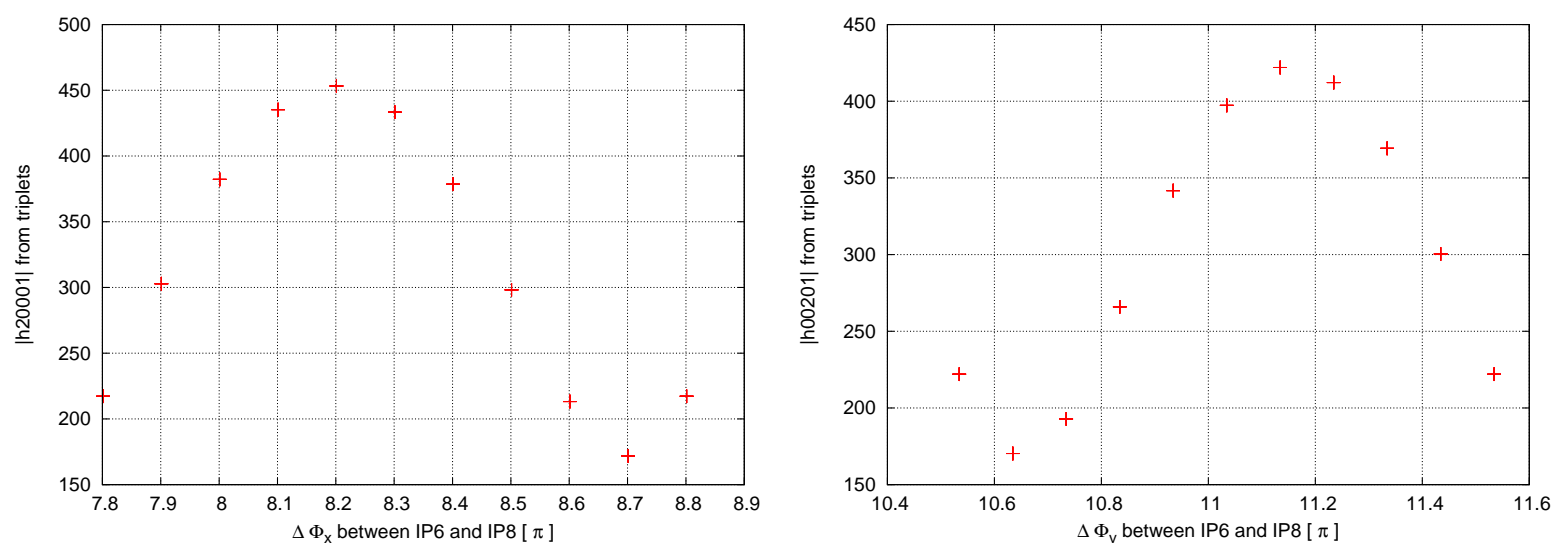

Figure 4: 2011pp-Yellow: RDT versus phase advances between IP6 and IP8.
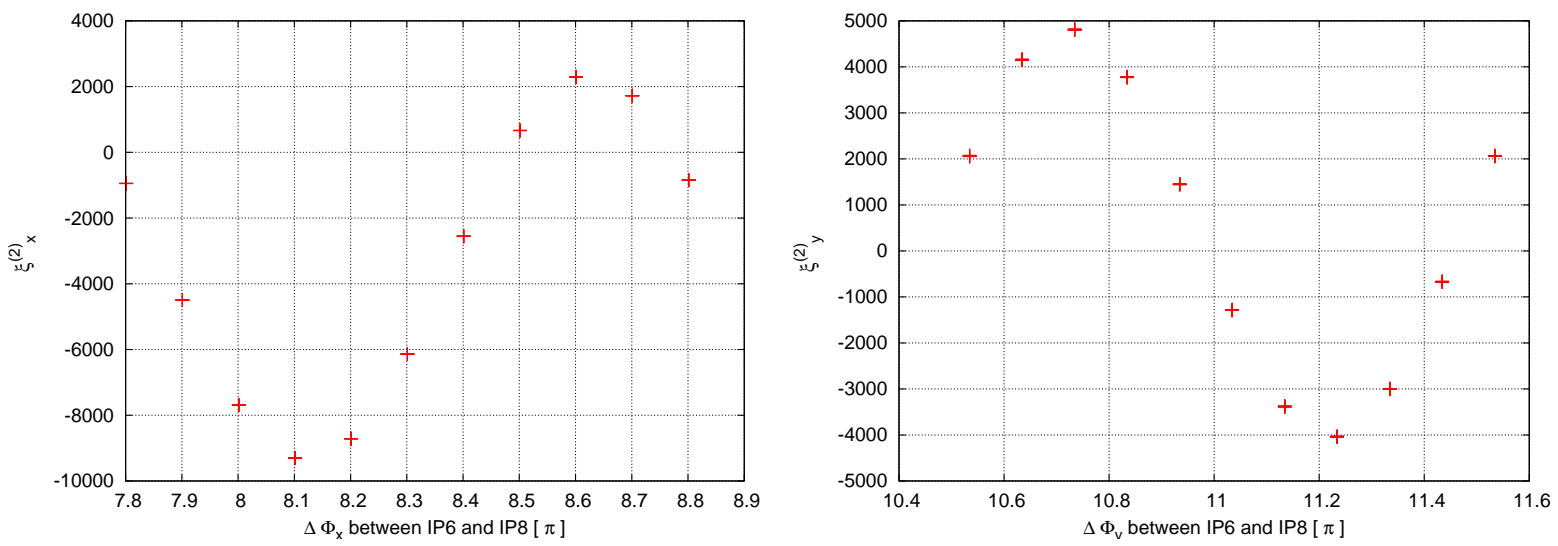

Figure 5: 2011pp-Yellow: second order chromaticities versus phase advances between IP6 and IP8.

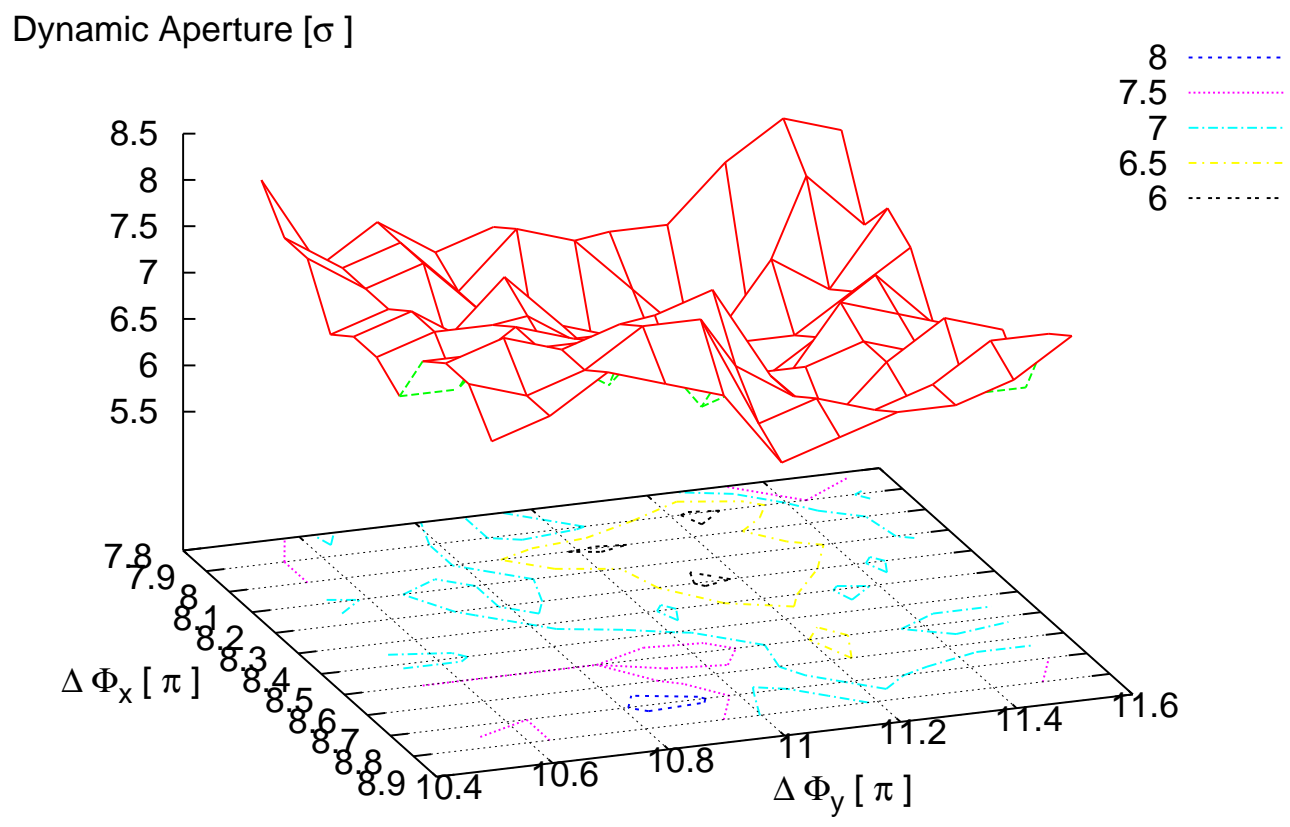

Figure 6: 2011pp-Yellow: Dynamic aperture versus phase advances between IP6 and IP8. 

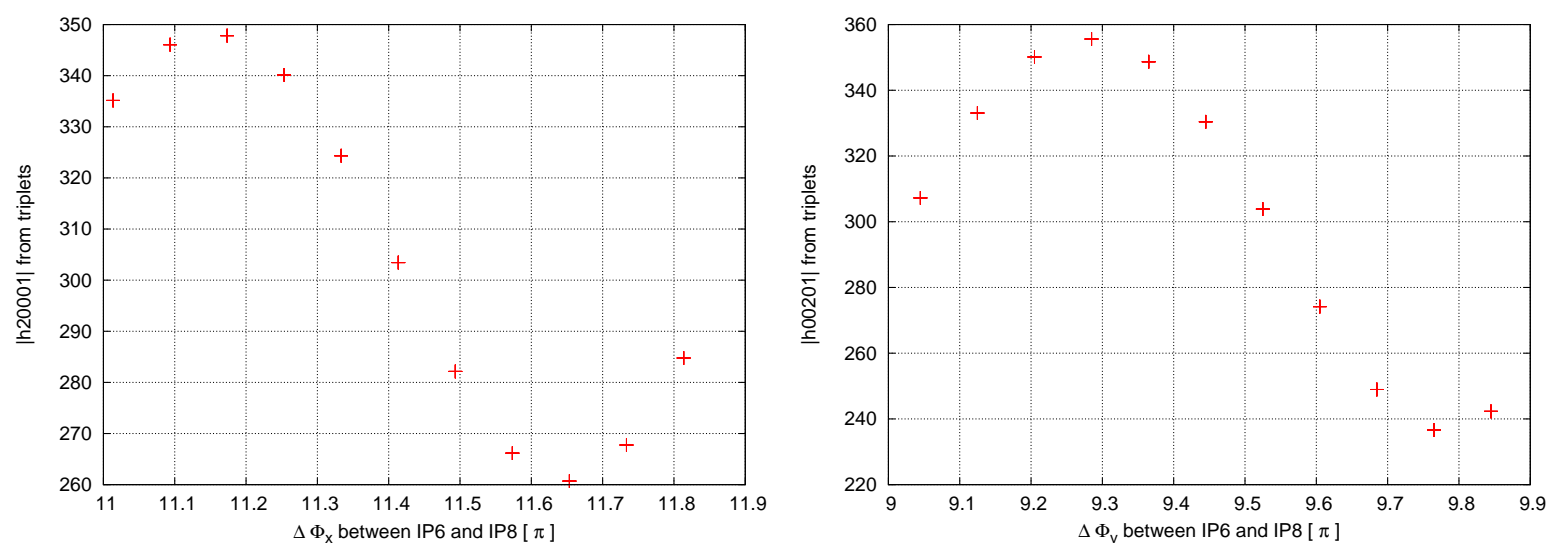

Figure 7: 2010Au-Blue: RDT versus phase advances between IP6 and IP8.
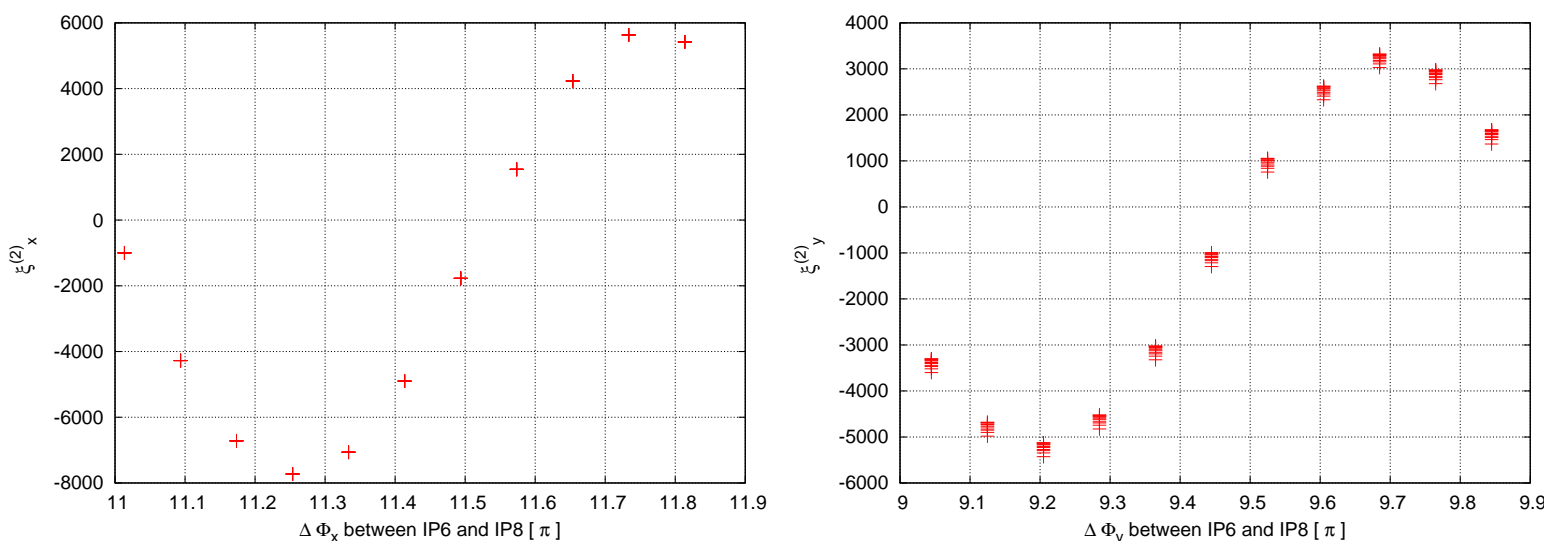

Figure 8: 2010Au-Blue: Second order chromaticities versus phase advances between IP6 and IP8.

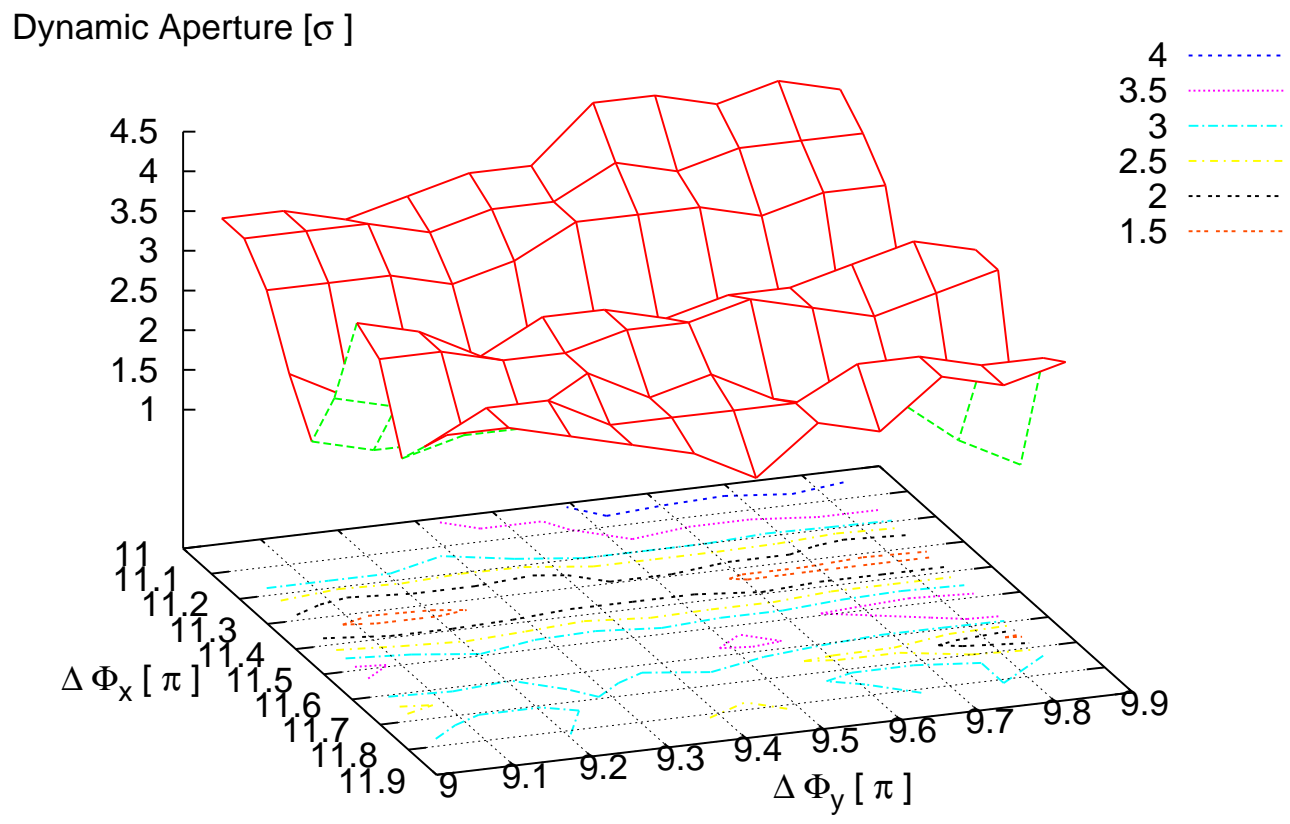

Figure 9: 2010Au-Blue: Dynamic aperture versus phase advances between IP6 and IP8. 

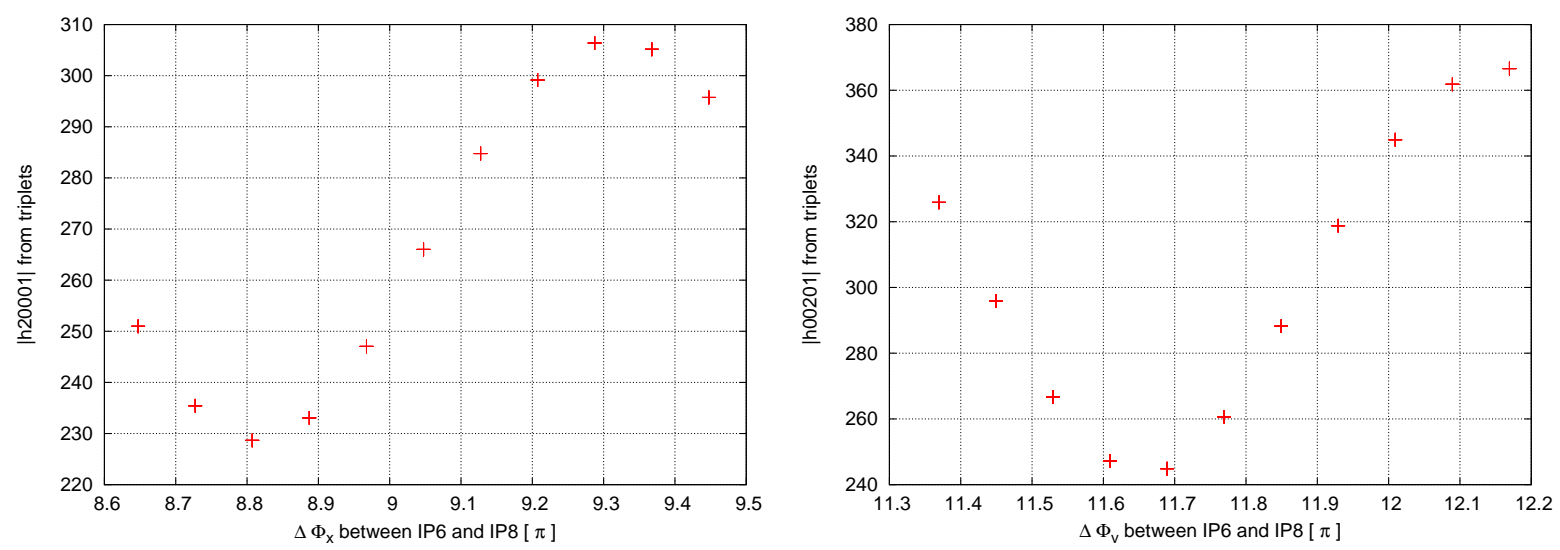

Figure 10: 2010Au-Yellow: RDT versus phase advances between IP6 and IP8.
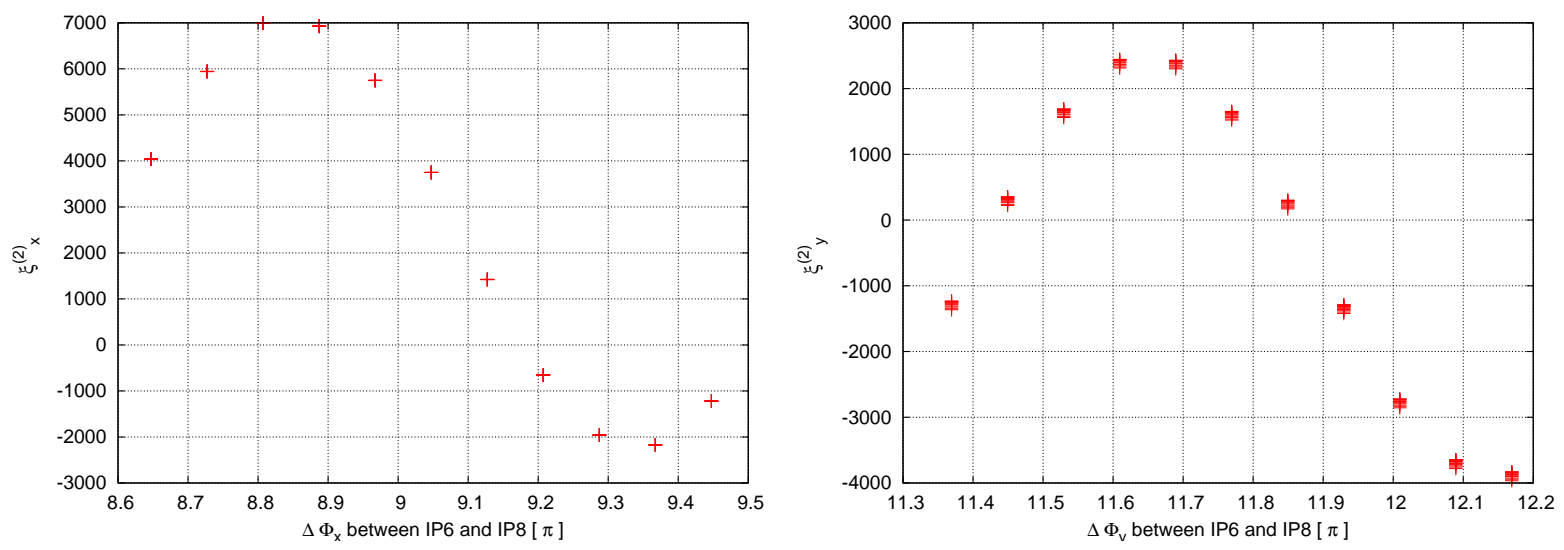

Figure 11: 2010Au-Yellow: Second order chromaticities versus phase advances between IP6 and IP8.

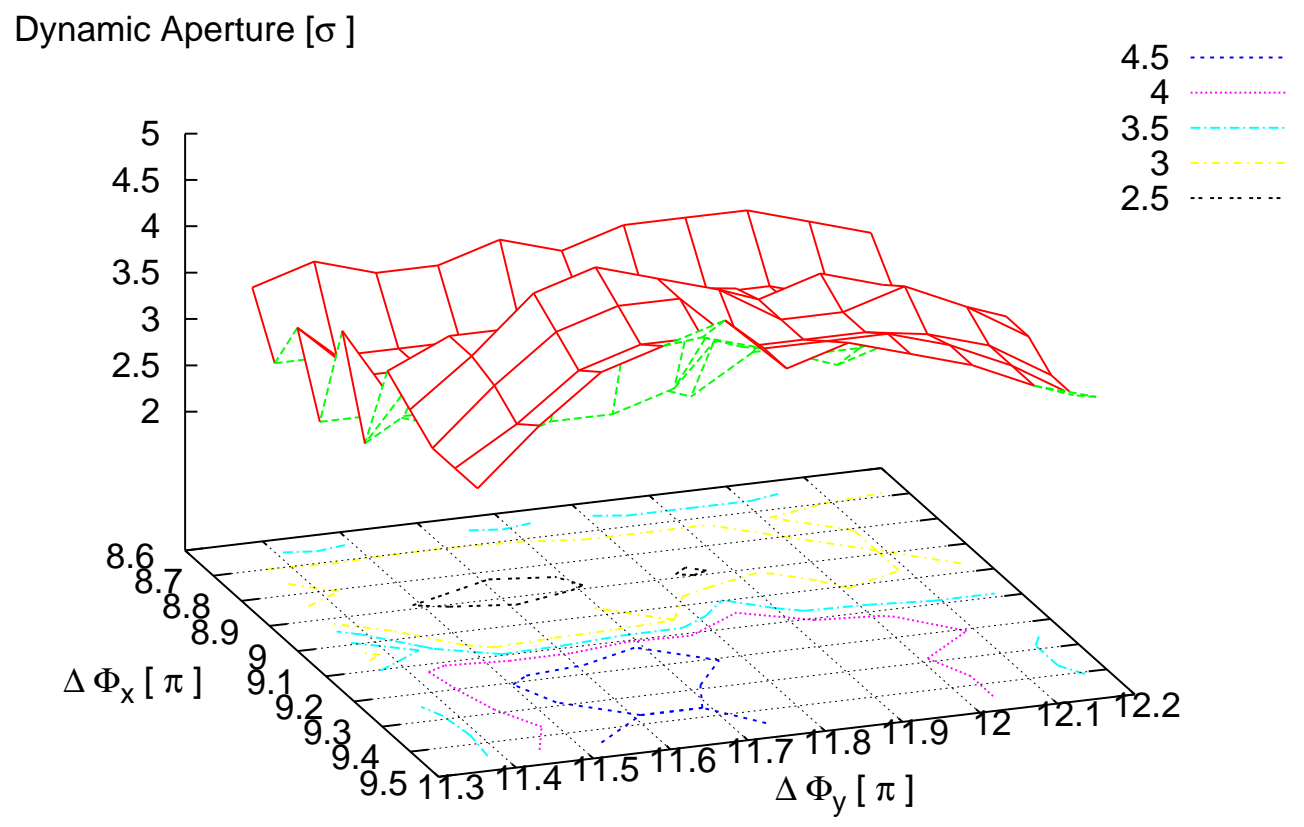

Figure 12: 2010Au-Yellow: Dynamic aperture versus phase advances between IP6 and IP8. 

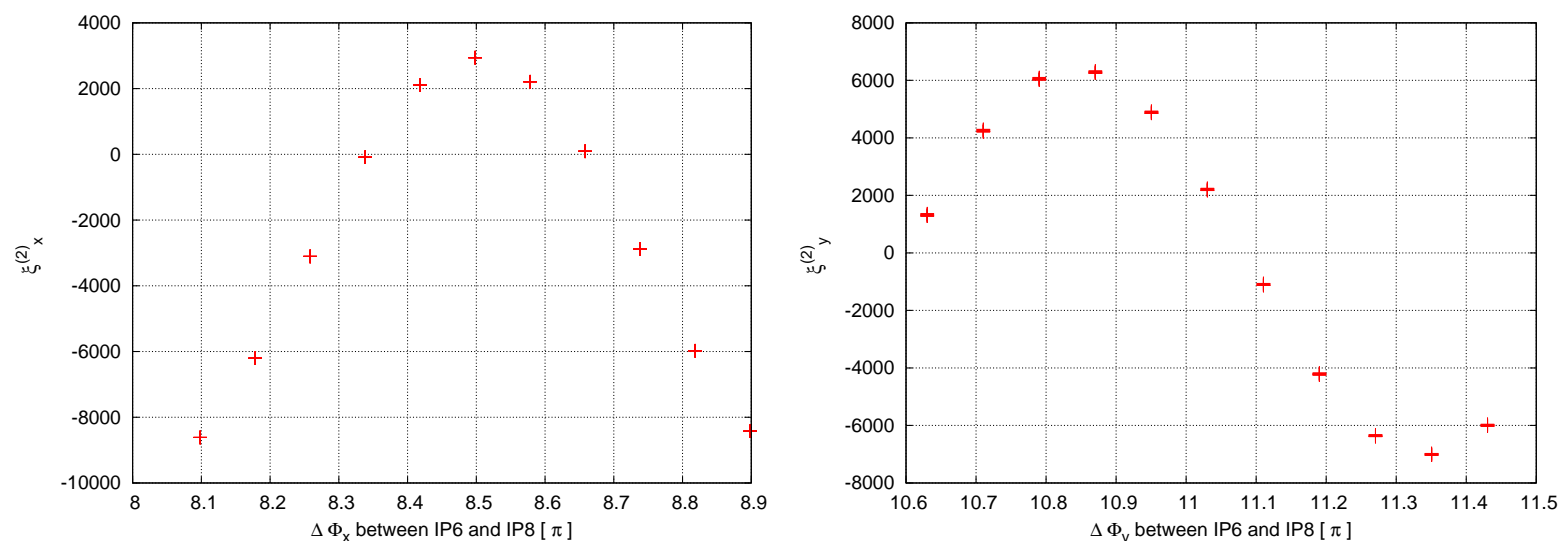

Figure 13: Elens lattice: Second order chromaticities versus phase advances between IP8 and e-lens.

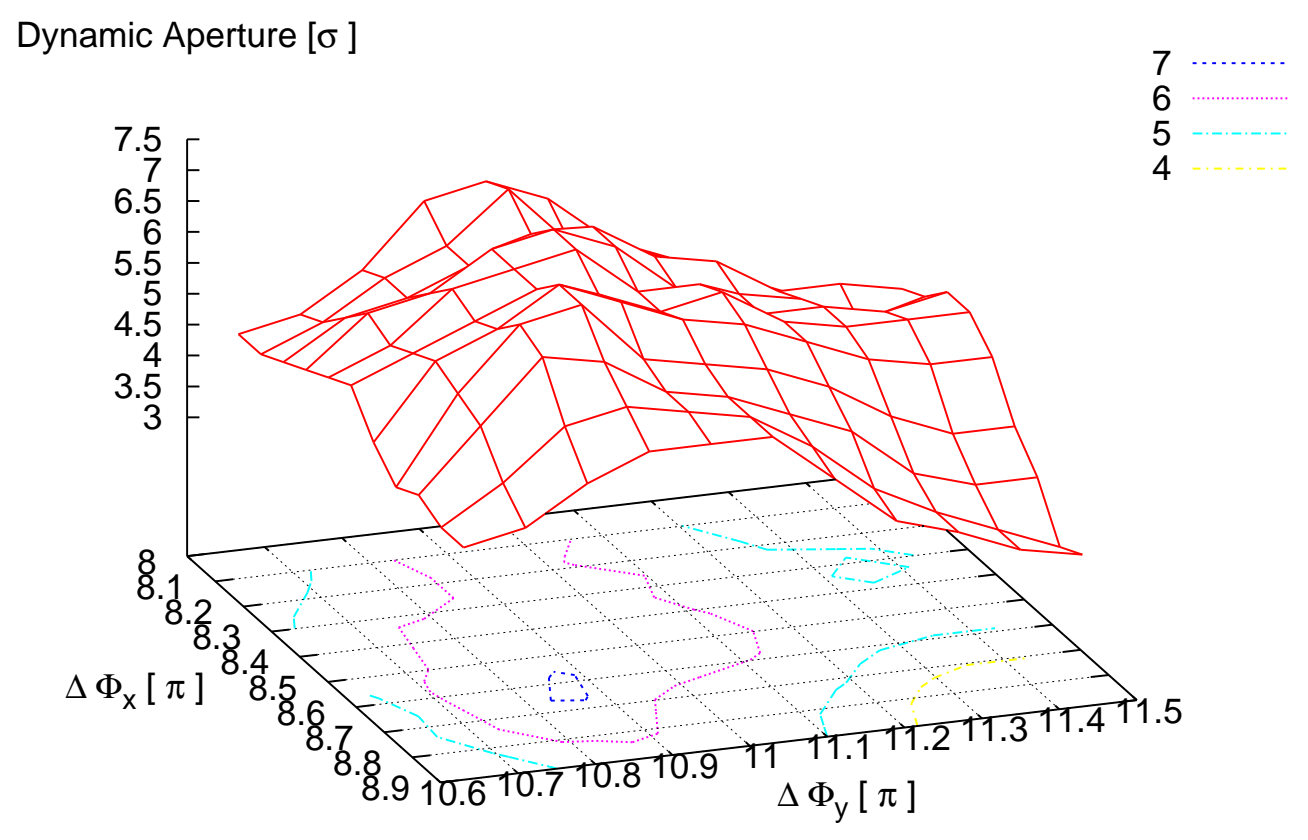

Figure 14: Elens lattice : Dynamic aperture versus phase advances between IP8 and e-lens. 